\title{
Prédiction de la réponse au remplissage vasculaire : que faire de tous ces indices proposés ?
}

\author{
Predicting fluid responsiveness: what to do with all these indices?
}

\section{De Backer}

Reçu le 31 octobre 2011 ; accepté le 30 janvier 2012

(C) SRLF et Springer-Verlag France 2012

Le remplissage vasculaire chez les patients de réanimation est encore un sujet d'intenses débats, tant sur le type de soluté de remplissage à utiliser que sur les modalités de son administration. Si le remplissage vasculaire permet d'améliorer la situation hémodynamique de certains patients via une augmentation du débit cardiaque [1] et de la perfusion tissulaire [2], l'administration excessive de perfusions liquidiennes peut également avoir des effets délétères $[3,4]$. Dans ce contexte, il semble légitime d'optimaliser l'administration de solutions de remplissage et d'éviter toute administration inutile de perfusions liquidiennes. Si tout le monde s'accorde sur ce principe, les moyens d'optimaliser la prescription du remplissage vasculaire sont multiples [5]. On peut utiliser des objectifs prédéfinis, la technique du fluid challenge et diverses techniques tendant à prédire la réponse au remplissage vasculaire.

L'utilisation d'objectifs prédéfinis, tels que l'obtention d'une pression veineuse centrale (PVC) à un niveau prédéfini est à proscrire, même si cette technique reste préconisée par certaines recommandations internationales [6]. En effet, l'augmentation de précharge est un moyen et non un but en soi. De plus, il est difficile d'en définir une valeur convenant à tous les patients (voire même à la majorité des patients), la réponse individuelle étant extrêmement variable, en dehors des situations caricaturales des niveaux très bas ou très élevés de PVC. Il en va de même pour les objectifs prédéfinis de surface ou volume des cavités cardiaques, comme on pourrait les mesurer par échocardiographie ou $\mathrm{PiCCO}^{\circledR}$ [7].

Le test de remplissage ou fluid challenge [8] est une technique qui administre une quantité définie de liquide sur une courte période de temps, en définissant des limites de sécurité à ne pas dépasser (augmentation de $\mathrm{PVC}$ de $2 \mathrm{mmHg}$ et/ou PVC max à $x \mathrm{mmHg}$ ) avec mesure des effets hémodyna-

\section{De Backer ( $\bowtie)$}

Service des soins intensifs médicochirurgicaux,

hôpital universitaire Erasme, université libre de Bruxelles,

808, route de Lennik, B-1070 Bruxelles, Belgique

e-mail : ddebacke@ulb.ac.be miques (débit cardiaque le plus souvent). Elle permet donc d'évaluer la réponse et la tolérance au remplissage vasculaire, mais pas d'en prédire la réponse hémodynamique a priori. Si l'utilisation parcimonieuse du fluid challenge est non délétère (car on arrête l'administration lorsque l'on constate que les derniers $250 \mathrm{ml}$ ne font plus d'effet), la répétition fréquente du test peut mener à l'excès d'administration de liquide. Tout récemment, une technique de mini-fluid challenge a été proposée : celle-ci consiste en l'administration d'un bolus de $100 \mathrm{ml}$ de liquide, avec évaluation de la réponse hémodynamique après une minute. Chez les 39 malades inclus dans l'étude préliminaire [9], cette technique semblait donner des résultats similaires au fluid challenge classique, mais une validation à plus grande échelle doit encore être réalisée. Notons que quelle que soit la manière utilisée pour prédire la réponse au remplissage, il est prudent d'administrer les liquides selon la technique du fluid challenge, car elle permet de s'assurer de la réponse réelle au remplissage (toutes les prédictions, aussi bonnes soient-elles, comportent un risque d'erreur) et surtout de la tolérance au remplissage. En effet, un patient peut augmenter son débit cardiaque lors d'une augmentation de précharge mais également élever dangereusement sa pression artérielle capillaire pulmonaire ou dilater son ventricule droit.

\section{Techniques de prédiction de la réponse au remplissage}

Afin d'éviter l'administration inutile de liquide, il semble souhaitable de prédire la réponse au remplissage. Toutes ces techniques utilisant des indices dynamiques se basent sur le fait qu'une modification transitoire de précharge soit observée naturellement, soit induite par une manœuvre externe, va générer une modification de débit cardiaque uniquement chez les patients qui sont sur la partie ascendante de la courbe de Starling, et qui vont donc répondre aux manœuvres de remplissage. Cette modification transitoire de 
précharge peut être observée, au cours d'un cycle respiratoire chez les patients sous ventilation mécanique, par une pause expiratoire prolongée chez les mêmes patients et par un test de lever de jambes passif chez tous les patients.

\section{Variations respiratoires de volume éjecté (et dérivées)}

De très nombreuses études ont montré que les variations de volume éjecté induites par la ventilation mécanique permettent de prédire la réponse au remplissage vasculaire [1]. On peut soit mesurer directement le volume éjecté par une technique permettant la mesure du débit cardiaque battement par battement, soit utiliser des mesures dérivées comme la pression artérielle pulsée. Bien que le déterminant physiologique de la mesure soit la variation de volume éjecté, les précisions respectives des mesures de débit et de pression artérielle font que le pouvoir diagnostique discriminant est meilleur pour le DeltaPP que pour les modifications respiratoires de volume éjecté. Dans une méta-analyse publiée en 2009, les courbes ROC étaient de 0,94 [0,92-0,96] pour le DeltaPP et $0,84[0,81-0,87]$ pour les variations de volume éjecté [10].

Pour autant que les prérequis soient présents, cette technique permet de prédire de manière extrêmement fiable la réponse au remplissage. Le problème majeur de cette technique est qu'elle nécessite que le patient soit en ventilation à pression positive avec un volume courant suffisant ( $\geq 8 \mathrm{ml} / \mathrm{kg}$ ) [11], une fréquence respiratoire pas trop élevée [12] et sans mouvement respiratoire spontané [13] (et donc souvent fortement sédaté), sans arythmie, sans dysfonction ventriculaire droite [14] et sans présenter d'augmentation de pression abdominale. $\mathrm{Vu}$ la tendance actuelle à limiter le volume courant et la sédation, le nombre de patients à qui cette technique s'applique tend à se restreindre. Notons au passage, qu'il n'est pas correct d'augmenter transitoirement le volume courant afin de réaliser les mesures à plus de $8 \mathrm{ml} / \mathrm{kg}$, car cela modifierait la précharge et la postcharge des deux ventricules et cette condition ne refléterait pas la situation obtenue lors du retour au volume courant préalable. Néanmoins, en dehors de ces conditions, la technique permet de prédire la réponse au remplissage avec une sensibilité de 0,89 (intervalle de confiance à $95 \%$ [IC $95 \%$ ] : 0,82-0,94) et spécificité de 0,88 (IC $95 \%$ : [0,81-0,92]) [10].

Où en est-on en 2012 ? Faut-il encore tenter de valider la variation respiratoire de volume éjecté avec une nouvelle technique de monitorage du débit cardiaque ? Les divers outils de monitorage du débit cardiaque ne peuvent que donner des résultats similaires, sauf si la technique de monitorage a un temps de réponse trop long ou est peu fiable dans les circonstances particulières de l'étude. L'intérêt de ce genre d'études est limité à un public très ciblé.

Il existe une tendance récente dans la littérature à tenter de valider des indices dérivés, obtenus de manière non invasive.
Est-ce souhaitable ? Selon moi, non. La mesure des variations de volume éjecté sans mesure de pression artérielle sanglante devient plus difficile. En effet, même si ces techniques, comme la mesure de la variation respiratoire de l'onde de pouls obtenue par phléthysmographie [15] ou photophléthysmographie [16], ont été validées, chez certains malades, souvent les plus graves, la qualité du signal est parfois insuffisante pour obtenir des mesures de qualité. L'utilisation de ce type de mesure totalement non invasive est donc limitée à la catégorie de malades les moins sévères. $\mathrm{Si}$ on peut encourager la tendance à approcher le malade de manière moins en moins invasive, il faut bien admettre que les malades les plus sévères (et donc ceux qui ont le plus besoin d'avoir une prédiction précise de leur réponse au remplissage) vont devoir bénéficier au minimum d'un monitorage sanglant de la pression artérielle, et on pourra dès lors calculer le DeltaPP.

De même, une mesure de la variation respiratoire du flux sanguin dans un organe donné ne peut également que donner des résultats similaires. Il est donc peu utile de continuer à vouloir montrer qu'une variation respiratoire de flux artériel d'un organe $x$ puisse prédire la réponse au remplissage.

\section{Variations respiratoires de diamètres des veines caves}

Bien que l'on étudie des fluctuations respiratoires, les implications et déterminants de celles-ci diffèrent en partie des fluctuations de volume éjecté. En effet, les fluctuations de diamètre de veine cave sont induites par les modifications de pression intrathoracique et affectées par la pression atriale droite qui reflète la balance entre la précharge et la fonction ventriculaire droite. Dans de petites études incluant des malades sélectionnés (absence de dysfonction ventriculaire gauche sévère), les variations respiratoires du diamètre des veines caves inférieure [17] et supérieure [18,19] ont montré un bon pouvoir diagnostique. Ces techniques, et la mesure de variation du diamètre de veine cave inférieure en particulier, connaissent un succès important car elles sont aisées à mesurer, rapidement disponibles et peu ou non invasives.

Notons cependant que les fluctuations de diamètre de veine cave et de volume éjecté du ventricule gauche peuvent être dissociées [12], et rien ne permet à ce stade de s'assurer qu'elles garderont une bonne valeur pronostique dans des cas d'insuffisance cardiaque gauche isolée. Bien que les fluctuations respiratoires de la veine cave inférieure puissent également être utilisées en ventilation spontanée, il existe un risque potentiel que les patients présentant de grands efforts respiratoires génèrent une fluctuation de diamètre de veine cave inférieure liée aux modifications cycliques de pression intra-abdominale plutôt que de pression intrathoracique. La réponse au remplissage serait alors moins prévisible. Ces divers indices doivent donc encore être validés dans des études plus larges et moins sélectionnées. 


\section{Test de lever de jambes passif}

Le test de lever de jambes passif est un autre test permettant d'obtenir une modification transitoire de précharge [20]. L'utilisation d'une mesure du débit cardiaque battement par battement est nécessaire, la simple mesure des modifications de pression pulsée étant moins discriminante [21]. Si ce test peut être utilisé chez les patients en ventilation spontanée ou présentant des arythmies, il présente cependant plusieurs limitations. D'une part, il faut s'assurer que la précharge est modifiée de façon suffisante. Pour ce faire, il faut non seulement lever les jambes mais également passer d'une position d'inclinaison du tronc de $30^{\circ}$ minimum à $0^{\circ}$ [22]. En effet, le lever des jambes ne mobilise que la quantité de sang contenue dans les veines capacitives des membres inférieurs, en l'associant à une bascule du tronc, cela permet également de mobiliser le sang contenu dans de grandes veines abdominales. D'autre part, il faut s'assurer de l'absence d'augmentation de pression intra-abdominale ou de l'usage de bas de contention. Même lorsque les conditions optimales semblent requises, les modifications de précharge peuvent être insuffisantes. Lakhal et al. [23] ont montré chez 110 patients que les performances du test étaient meilleures chez les patients présentant une augmentation de PVC d'au moins $2 \mathrm{mmHg}$ que chez ceux qui ne présentaient pas de modification de PVC. De manière globale, les performances du test sont excellentes, comme l'atteste une méta-analyse de Cavallaro et al. [24]. Notons cependant que celle-ci incluait 178 patients et n'a pas pris en compte les études moins favorables de Mahjoub et al. [25] (41 patients) et de Lakhal et al. [23] (102 patients). Cette méta-analyse devrait donc être actualisée.

\section{Pause expiratoire}

Le test de pause expiratoire se base sur le fait que l'on induit une autotransfusion de sang des compartiments périphériques par diminution transitoire de la pression intrathoracique. Lors de la ventilation mécanique, l'obstacle au retour veineux est représenté par la pression intrathoracique moyenne sur un cycle respiratoire. Lors de l'application d'une pause expiratoire, la pression intrathoracique moyenne est brutalement abaissée au niveau de la pression de fin d'expiration, ce qui facilite l'entrée du sang veineux dans le thorax. Cette pause doit être suffisamment prolongée pour permettre une modification suffisante de précharge. Une étude a validé ce concept jusqu'à présent [26]. Son intérêt particulier réside dans le fait que des patients présentant une arythmie peuvent être analysés. Par contre, l'impact des autres limitations (bas volume courant, dysfonction ventriculaire droite, pression abdominale élevée...), voire de l'impact réel sur la précharge ventriculaire, n'a pas été étudié. Ce test mérite plus ample validation et sa place reste à déterminer.

\section{Que faut-il faire en 2012 ? Quels axes méritent d'être investigués ?}

Comme nous l'avons vu en détail plus haut, la prédiction de la réponse au remplissage repose sur une base physiologique solide et a été, en tout cas pour les variations respiratoires de volume éjecté et de pression pulsée, amplement validée. Il y a donc peu de place pour des études supplémentaires dans ce domaine, sauf si une approche totalement innovatrice venait à être proposée. Cependant, les validations ont toutes été développées a posteriori, c'est-à-dire en évaluant quelles variables, et à quel seuil, prédisaient mieux la réponse au remplissage. À ce stade, il faut bien admettre qu'une validation externe manque toujours, personne n'ayant vérifié la pertinence des seuils définis dans la littérature sur une nouvelle cohorte de patients.

Plus important sans doute, il faut bien admettre que ces seuils ne correspondent pas à une valeur en dessous de laquelle aucun patient ne répond et au-dessus tous les patients répondent au remplissage. Le concept de zone d'incertitude, ou zone grise, doit donc être accepté (Fig. 1). Cela a été récemment développé dans une étude élégante conduite par Cannesson et al. [27]. Ces auteurs ont étudié une série de 413 patients (quatre centres). La zone grise a été définie comme correspondant à l'IC $95 \%$ autour du point correspondant au meilleur compromis entre la spécificité et la sensibilité. De manière alternative, ils ont statué que l'on pouvait admettre un niveau d'incertitude diagnostique de $10 \%$. Dans ce contexte, tous les points correspondant à un

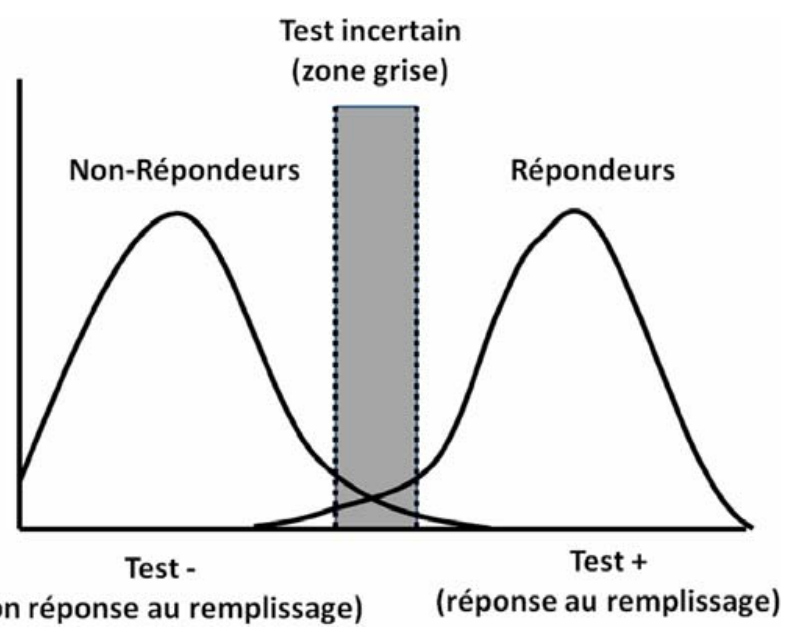

Fig. 1 Concept de la zone grise. Ce diagramme représente la distribution de la fréquence de la valeur d'un test chez les répondeurs et les non-répondeurs au remplissage. Le seuil du test, déterminé par courbe ROC (receiver operating characteristics), correspond à l'intersection des deux histogrammes. Comme il existe un certain chevauchement, il existe un degré d'incertitude autour de ce seuil, ou zone grise, dans laquelle les résultats du test sont incertains 
niveau de sensibilité ou de spécificité inférieur à $90 \%$ avaient été inclus dans cette zone grise. Les deux approches statistiques ont fourni des résultats similaires. Cette zone grise, correspondant à un DeltaPP compris entre 9 et $13 \%$, incluait $24 \%$ du collectif étudié. Chez ces patients, les chances de réponse au remplissage étaient de $50 \%$, soit équivalentes au lancer d'une pièce de monnaie... De nouvelles études devraient donc évaluer ce qu'il y a lieu de faire dans ce contexte : faut-il accepter cette incertitude ou faut-il implémenter un second test (lever de jambes? Pause ? Veine cave ?) pour affiner la prédiction.

Enfin faut-il évaluer l'intérêt de telles pratiques sur le devenir du patient? En d'autres termes ne complique-t-on pas inutilement nos pratiques pour un résultat assez minime (voire nul) sur le devenir de nos patients ? Pour tester cela, il $\mathrm{y}$ a quatre grandes questions à explorer.

Primo, quelle est la place exacte de ces diverses techniques en pratique courante ? En d'autres termes peut-on les utiliser chez la plupart des malades ou ces techniques sont-elles restreintes à une minorité de malades ? Dans de nombreux cas, le test ne peut être réalisé car non valide (exemple : DeltaPP en ventilation spontanée) ou la manœuvre est potentiellement dangereuse (exemple : lever de jambes chez un patient en ventilation spontanée qui présente une occlusion intestinale). Il faudrait donc évaluer quelle est l'utilisation réelle en pratique clinique des techniques utilisées pour prédire et guider la réponse au remplissage. Une telle étude sera prochainement réalisée par le groupe de recherche de la Société européenne de soins intensifs (étude FENICE, par l'ECCRN Trial Group). Cela permettra de connaître quels sont les indices utilisés et quel est le potentiel de progression de ces divers indices dynamiques.

Secundo, quel test utiliser? Si la validation des variations de volume éjecté/pression pulsée est assez extensive, celle du test de lever de jambes et surtout de l'évaluation des diamètres cave nécessite un plus grand niveau de preuve. En particulier, beaucoup de cliniciens sélectionnent tel ou tel test pour des raisons qui tiennent parfois plus à des choix personnels qu'aux qualités intrinsèques des tests ou aux spécificités des malades. Il serait intéressant de comparer la valeur pronostique des divers tests dans une relativement large série, afin d'établir une hiérarchie diagnostique et d'évaluer l'intérêt du cumul de divers tests autour de leurs zones grises respectives.

Tertio, le seuil utilisé pour définir la réponse au remplissage est-il adéquat? La plupart des études définissent celleci comme une augmentation de $10-15 \%$ du débit cardiaque. À quoi correspond ce seuil ? Il a été défini comme deux fois l'erreur de la mesure du débit cardiaque, ainsi, il y a peu de risque que l'erreur de mesure puisse expliquer les modifications observées. Si des techniques plus fiables de mesure du débit cardiaque venaient à être développées, ce seuil pourrait être revu à la baisse. Si l'erreur sur la mesure du débit cardiaque était proche de zéro, il faudrait définir ce seuil comme l'augmentation minimale pouvant avoir un impact clinique. Dans ce contexte, il se pourrait que l'on puisse également parler en différence absolue plutôt que relative (une augmentation de débit cardiaque $0,45 \mathrm{l} / \mathrm{min}$ correspond à une augmentation de $15 \%$ si le débit cardiaque de base était à $3 \mathrm{l} / \mathrm{min}$ mais seulement $9 \%$ s'il était à $5 \mathrm{l} / \mathrm{min}$ ). On pourrait également se demander si la réponse au remplissage vasculaire doit réellement s'exprimer en termes d'augmentation du débit cardiaque. En effet, certaines études ont montré qu'il pouvait y avoir chez certains patients une dissociation entre la réponse microcirculatoire, reflétant peutêtre mieux la perfusion tissulaire, et les modifications de débit cardiaque en réponse au remplissage vasculaire [2]. Enfin, lorsque nous aurons les réponses à ces questions, nous pourrons nous pencher sur la question finale : cela changet-il le devenir du patient ? Bien que la question soit intéressante, il faut craindre que la réponse soit difficile à apporter. Ces études, comparant deux pratiques, risquent d'être polluées par les habitudes du service/médecin (comment négliger chez un patient du groupe témoin une grande variation de pression artérielle détectée sur le moniteur?). De plus, il sera difficile de s'assurer qu'une indication de remplissage vasculaire (DeltaPP fortement positif chez un patient du groupe interventionnel) n'a pas été manquée par l'équipe prenant en charge le patient. Il y a dès lors fort à parier que les deux groupes seront, in fine, traités de manière fort similaire.

En attendant la réponse à ces diverses questions, que faire en 2012 sur le plan pratique ? Mon approche personnelle peut se résumer comme suit (Fig. 2). Si le patient présente les prérequis pour la mesure du DeltaPP, celui-ci sera privilégié car il est en effet validé par le plus grand nombre d'études, est le plus simple à mesurer et probablement soumis au

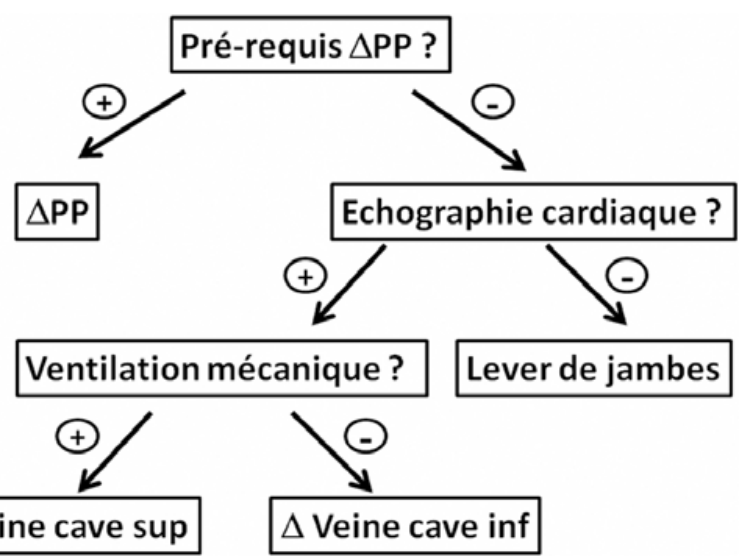

Fig. 2 Proposition pratique de l'auteur concernant l'utilisation des divers tests. Voir texte pour explications. DeltaPP représente les variations respiratoires de pression pulsée, $\Delta$ veine cave supérieure et $\Delta$ veine cave inférieure les variations respiratoires de diamètre des veines caves supérieure et inférieure 
risque d'erreur le moins important. Si ces prérequis ne sont pas présents, l'approche échographique sera privilégiée. En dehors de l'évaluation de la précharge-dépendance, celle-ci permet également d'évaluer d'autres aspects de la fonction cardiaque. En ventilation mécanique, la mesure de la variation de diamètre de la veine cave supérieure est préférée à celle de la veine cave inférieure, car moins influencée par des facteurs confondants comme par exemple une hypertension abdominale. Lorsque l'échographie cardiaque n'est pas réalisable, le lever de jambes peut être considéré. Enfin, n'oublions pas qu'en cas de doute ou d'incapacité à réaliser le test, une épreuve de remplissage peut être réalisée.

\section{Conclusion}

Nous disposons actuellement de divers outils qui nous permettent de prédire la réponse au remplissage vasculaire. Ces outils doivent cependant être appliqués avec discernement, en en reconnaissant les limitations. Diverses questions restent à élucider et, en particulier, que faire lorsque la réponse au test prédictif se situe dans la zone d'incertitude. Enfin, quelle que soit la façon dont on prédit la réponse au remplissage, il est prudent d'administrer les liquides selon la technique du fluid challenge, afin de s'assurer de l'efficacité et de la tolérance du remplissage vasculaire.

Conflit d'intérêt : l'auteur a reçu un financement et du matériel de recherches par Ewards Lifesciences et Vytech.

\section{Références}

1. Michard F, Boussat S, Chemla D, et al (2000) Relation between respiratory changes in arterial pulse pressure and fluid responsiveness in septic patients with acute circulatory failure. Am J Respir Crit Care Med 162:134-8

2. Ospina-Tascon G, Neves AP, Occhipinti G, et al (2010) Effects of fluids on microvascular perfusion in patients with severe sepsis. Intensive Care Med 36:949-55

3. Wiedemann HP, Wheeler AP, Bernard GR, et al (2006) Comparison of two fluid-management strategies in acute lung injury. $\mathrm{N}$ Engl J Med 354:2564-75

4. Sakr Y, Vincent JL, Reinhart K, et al (2005) High tidal volume and positive fluid balance are associated with worse outcome in acute lung injury. Chest 128:3098-108

5. Marik PE, Monnet X, Teboul JL (2011) Hemodynamic parameters to guide fluid therapy. Ann Intensive Care 1:1

6. Dellinger RP, Levy MM, Carlet JM, et al (2008) Surviving Sepsis Campaign: international guidelines for management of severe sepsis and septic shock. Intensive Care Med 34:17-60

7. Muller L, Louart G, Bengler C, et al (2008) The intrathoracic blood volume index as an indicator of fluid responsiveness in critically ill patients with acute circulatory failure: a comparison with central venous pressure. Anesth Analg 107:607-13

8. Vincent JL, Weil MH (2006) Fluid challenge revisited. Crit Care Med 34:1337

9. Muller L, Toumi M, Bousquet PJ, et al (2011) An increase in aortic blood flow after an infusion of $100 \mathrm{ml}$ colloid over 1 minute can predict fluid responsiveness: the mini-fluid challenge study. Anesthesiology 115:541-7

10. Marik PE, Cavallazzi R, Vasu T, Hirani A (2009) Dynamic changes in arterial waveform derived variables and fluid responsiveness in mechanically ventilated patients: a systematic review of the literature. Crit Care Med 37:2642-7

11. De Backer D, Heenen S, Piagnerelli M, et al (2005) Pulse pressure variations to predict fluid responsiveness: influence of tidal volume. Intensive Care Med 31:517-23

12. De Backer D, Taccone FS, Holsten R, et al (2009) Influence of respiratory rate on stroke volume variation in mechanically ventilated patients. Anesthesiology 110:1092-7

13. Heenen S, De Backer D, Vincent JL (2006) How can the response to volume expansion in patients with spontaneous respiratory movements be predicted? Crit Care 10:R102

14. Mahjoub Y, Pila C, Friggeri A, et al (2009) Assessing fluid responsiveness in critically ill patients: False-positive pulse pressure variation is detected by Doppler echocardiographic evaluation of the right ventricle. Crit Care Med 37:2570-5

15. Biais M, Stecken L, Ottolenghi L, et al (2011) The ability of pulse pressure variations obtained with CNAP device to predict fluid responsiveness in the operating room. Anesth Analg 113:523-8

16. Cannesson M, Attof Y, Rosamel P, et al (2007) Respiratory variations in pulse oximetry plethysmographic waveform amplitude to predict fluid responsiveness in the operating room. Anesthesiology 106:1105-11

17. Vieillard-Baron A, Chergui K, Rabiller A, et al (2004) Superior vena caval collapsibility as a gauge of volume status in ventilated septic patients. Intensive Care Med 30:1734-9

18. Feissel M, Michard F, Faller JP, Teboul JL (2004) The respiratory variation in inferior vena cava diameter as a guide to fluid therapy. Intensive Care Med 30:1834-7

19. Barbier C, Loubieres Y, Schmit C, et al (2004) Respiratory changes in inferior vena cava diameter are helpful in predicting fluid responsiveness in ventilated septic patients. Intensive Care Med 30:1740-6

20. Boulain T, Achard JM, Teboul JL, et al (2002) Changes in BP induced by passive leg raising predict response to fluid loading in critically ill patients. Chest 121:1245-52

21. Monnet X, Rienzo M, Osman D, et al (2006) Passive leg raising predicts fluid responsiveness in the critically ill. Crit Care Med $34: 1402-7$

22. Jabot J, Teboul JL, Richard C, Monnet X (2009) Passive leg raising for predicting fluid responsiveness: importance of the postural change. Intensive Care Med 35:85-90

23. Lakhal K, Ehrmann S, Runge I, et al (2010) Central venous pressure measurements improve the accuracy of leg raising-induced change in pulse pressure to predict fluid responsiveness. Intensive Care Med 36:940-8

24. Cavallaro F, Sandroni C, Marano C, et al (2010) Diagnostic accuracy of passive leg raising for prediction of fluid responsiveness in adults: systematic review and meta-analysis of clinical studies. Intensive Care Med 36:1475-83

25. Mahjoub Y, Touzeau J, Airapetian N, et al (2010) The passive leg-raising maneuver cannot accurately predict fluid responsiveness in patients with intra-abdominal hypertension. Crit Care Med 38:1824-9

26. Monnet X, Osman D, Ridel C, et al (2009) Predicting volume responsiveness by using the end-expiratory occlusion in mechanically ventilated intensive care unit patients. Crit Care Med 37:951-6

27. Cannesson M, Le Manach Y, Hofer CK, et al (2011) Assessing the diagnostic accuracy of pulse pressure variations for the prediction of fluid responsiveness: a "gray zone" approach. Anesthesiology 115:231-41 